Ann. Génét. Sél. anim., 1972, 4 (3), 45I-467.

\title{
LES PARAMETRES GÉNÉTIQUES DES CARACTERES D'ENGRAISSEMENT ET DE CARCASSE CHEZ LE PORC DE PIETRAIN
}

\author{
R. HANSET et G. VAN SNICK * \\ Faculté de Médecine vétérinaive \\ (Université de Liège) \\ Cureghem-Bruxelles \\ * Service de l'Élevage, \\ Ministère de l'Agriculture \\ Bruxelles
}

RÉSUMÉ

Dans cette étude, on analyse les données recueillies en stations sur I 087 sujets de race de Piétrain, issus de 120 verrats et de 317 truies.

Les effets du sexe et des variations du poids abattu sur les caractères d'engraissement et de carcasse sont étudiés.

Les paramètres génétiques, coefficients d'héritabilité et corrélations génétiques, sont estimés pour I 3 caractères à partir des composantes paternelles de la variance.

La corrélation génétique entre le gain quotidien moyen et la proportion de morceaux maigres dans la carcasse est négative et significativement différente de zéro.

La tendance annuelle observée au niveau des moyennes est étudiée. Dans une station, elle est hautement significative pour la proportion de morceaux maigres dans la carcasse. L'amélioration de ce caractère est parallèle dans les deux sexes (absence d'interaction sexe $\times$ année) ; elle s'accompagne d'une diminution de l'importance relative de l'épaule par rapport aux autres morceaux maigres (carré et jambon).

\section{I. - INTRODUCTION}

Malgré le manque d'informations précises concernant l'origine du porc de $P i e ́$ train, il est un fait que cette nouveauté génétique ne pouvait survivre et se multiplier que dans un environnement favorable, c'est-à-dire en l'occurrence, dans un contexte commercial qui l'avantageait, par rapport aux types génétiques contemporains de son apparition. 
En Belgique, en effet, le marché des animaux de boucherie, qu'il s'agisse des bovins ou des porcs, a cette particularité qu'il discrimine, comme nulle part ailleurs, les différences de conformation. La valeur commerciale d'un sujet de boucherie n'est pas une fonction linéaire mais curviligne du pourcentage de viande dans la carcasse. On a pu montrer que, chez le porc en particulier, la valeur unitaire des différents morceaux était d'autant plus élevée que la carcasse était plus riche en viande (HANSET, I97I).

Le début de la notoriété du porc de Piétrain, dans les diverses régions du pays d'abord, au-delà de nos frontières ensuite, remonte à une époque (I950-5I) où le marché porcin belge était particulièrement difficile et où la différence de prix au $\mathrm{kg}$ vif entre un Piétrain et un sujet d'une autre race pouvait atteindre ro francs belges et plus. Il est bien connu, en économie, que les prix des différentes qualités d'un même produit ont tendance à s'écarter, lorsque les prix sont en baisse. Bref, les conditions étant remplies. le Piétrain se multiplia et se répandit.

La sélection fut organisée dans sa province d'origine d'abord (le Brabant), dans le reste du pays, par la suite. Elle empruntait les structures déjà mises en place pour les autres races reconnues en Belgique et comportait, en particulier, le testage en station. De ce testage en station, les éleveurs de porc de Piétrain n'ont pas fait 1'usage qu'on aurait pu espérer. C'est pourquoi, malgré la grosse capacité de testage dont est doté notre pays, les données relatives au Piétrain sont assez limitées.

\section{II. - MATÉRIEL ET MÉTHODES}

\section{I. - Matériel animal}

Les données se rapportent à des lots testés dans deux stations (Wavre et Marloie), à loges individuelles, de l'année 196r à l'année 1970. N'ont été retenues que les données se rapportant à des sujets dont le poids final tombait entre les limites $90 \mathrm{~kg} \pm \mathrm{Io} \mathrm{kg}$ et dont le père était représenté par deux lots au moins. Après cette sélection, il restait I 087 sujets $(560$ mâles et 527 femelles), provenant de I 20 verrats et 317 truies. Les lots sont constitués, au départ, de deux castrats et deux femelles, amenés à la station au poids approximatif de $20 \mathrm{~kg}$. Ils subissent d'abord une quarantaine pendant laquelle ils sont vermifugés et vaccinés.

L'épreuve commence au poids de $25 \mathrm{~kg}$ et se termine au poids de $90 \mathrm{~kg}$. Les animaux reçoivent, en trois repas, une alimentation humide semi ad. libitum, c'est-à-dire avec ajustement selon l'appétit. En fait, comparativement à une alimentation réellement ad. libitum, le système appliqué dans les stations de sélection belges apporte une certaine restriction de l'alimentation, évaluée à I 2,9 p. Ioo (De Wilde et VAn Schoubroek, 1970). A partir de 1965, la formule alimentaire utilisée dans les stations belges a été modifiée (tabl. I). Le matériel a été divisé en conséquence. La période I (6I-65) se rapporte au premier type de ration et comporte 50 verrats et $\mathrm{I} 22$ truies ; la période II (66-70) se rapporte au second type de ration et comporte 70 verrats et 195 truies.

Le poids vif final est déterminé, à jeun, le lundi matin. Le chargement a lieu le mardi matin. Après un séjour en chambre froide, une demi-carcasse est découpée, selon la découpe bruxelloise (fig. I). L'ensemble des morceaux maigres ou "Maigre total " comprend : le carré, le jambon, I'épaule, les déchets maigres. L'ensemble des morceaux gras ou "Gras total " comprend : lard dorsal, parois ventrales, saindoux, collier. Le rendement à l'abattage s'obtient en divisant le poids de la carcasse froide (deux fois le poids d'une demi-carcasse froide) par le poids vif final mesuré à jeun à la station. La longueur de la carcasse entre la première côte et le pubis est prise : sur la demi-carcasse suspendue. L'épaisseur du lard est mesurée à trois endroits : épaule, dos et rein.

Le gain quotidien est donné par la relation : (poids vif final - poids initial)/durée du test ; U'indice de consommation est donné par : quantité consommée/(poids final - poids initial). 
TABLEAU I

Formules alimentaires utilisées dans les stations belges

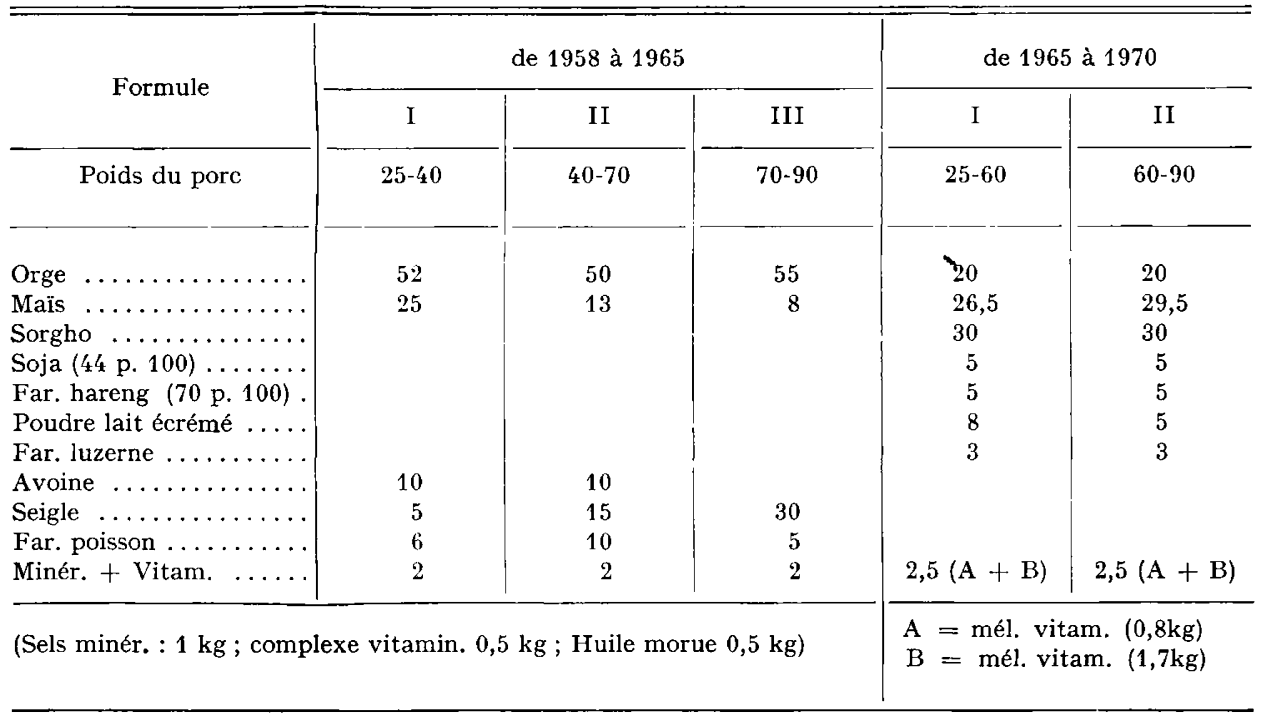

$\mathrm{A}=$ mélange vitaminique : vit. A $312500 \mathrm{UI}$; vit. $\mathrm{D}_{3} 125000 \mathrm{UI}$; vit. $\mathrm{B}_{2} 80 \mathrm{mg}$; ac. pantothén. $625 \mathrm{mg}$; niacine $1250 \mathrm{mg}$; vit. E $2500 \mathrm{mg}$; oxytétracycl. $1250 \mathrm{mg}$; éthoxyguin $1250 \mathrm{mg}$ par kilo; milocorn ad. $100 \mathrm{~kg}$.

$\mathrm{B}=$ mélange minéral : $\mathrm{MnSO}_{4}, 5 \mathrm{H}_{2} \mathrm{O} 1$ p. $100 ; \mathrm{ZnSO}_{4}, 7 \mathrm{H}_{2} \mathrm{O} 2$ p. $100 ; \mathrm{FeSO}_{4}, 7 \mathrm{H}_{2} \mathrm{O} 1$ p. $100 ; \mathrm{CuSO}_{4}$, $5 \mathrm{H}_{2} \mathrm{O} 4,5$ p. $100 ; \mathrm{CoSO}_{4}, 7 \mathrm{H}_{2} \mathrm{O} 0,03$ p. $100 ; \mathrm{I} 0,0015$ p. $100 ; \mathrm{NaCl} 12$ p. 100 ; $\mathrm{CaCO} 19,45$ p. 100 , CaHPO 60 p. 100 .

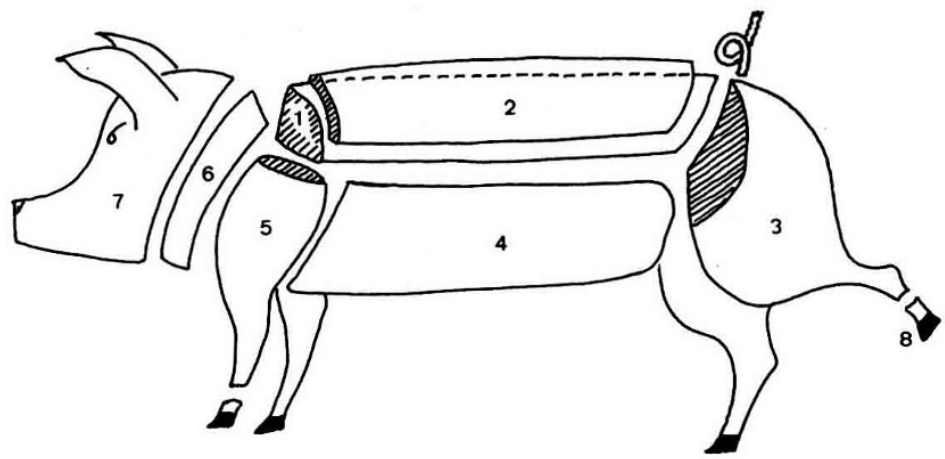

FIG. I. - Découpe bruxelloise
I. Carré ou longe
2. Lard dorsal
5. Épaule
3. Jambon
6. Collier
4. Paroi ventrale
7. Tête
8. Pieds 


\section{2. - L'estimation des paramètres génétiques $\left(h^{2}, r g\right)$}

Les données ont été analysées selon la méthode d'analyse de variance et de covariance à classification hiérarchique : stations - années — pères — mères.

A partir des espérances des sommes de carrés et de produits fournies par les analyses de variance et de covariance, on peut estimer les composantes de variance et de covariance nécessaires à l'estimation des paramètres génétiques. La méthode suivie a été exposée par de nombreux auteurs : King et Henderson (I954), Kempthorne (I957), Dickerson (I96o), Le Roy (I960, I966).

Des croisements au hasard dans une grande population panmictique impliquent I'absence de parenté $(\mathrm{R}=\mathrm{o})$ entre femelles croisées à un même mâle et entre femelles et mâles croisés entre eux (consanguinité des produits). Les éleveurs évitent la consanguinité. Ils évitent moins à faire tester des lots provenant d'un verrat et de truies apparentées (propres sœurs, demi-sœurs), malgré une réglementation tendant à limiter cette pratique.

Dans le cas d'une parenté moyenne, $R$, (coefficient de WrIGHT (I922) = deux fois le coefficient de parenté de MALÉcot (I948), en l'absence de consanguinité) entre femelles croisées au même mâle mais avec parenté nulle entre mâles ou entre mâle et femelle d'un couple, avec en outre variances de dominance et d'épistasie nulles, la variance génétique additive $\sigma_{\mathbf{A}}^{2}$ s'estime par :

$$
4 \cdot \widehat{\sigma}_{s}^{2} /(\mathrm{I}+\mathrm{R}) \quad \text { plutôt que par } 4 \widehat{\sigma}_{s}^{2}
$$

$\left(\hat{\sigma}_{s}^{2}=\right.$ composant de variance paternel)

En effet, $\sigma_{s}^{2}$ (le paramètre estimé par $\widehat{\sigma}_{s}^{2}$ ) n'est plus égal à $\frac{\mathrm{I}}{4} \sigma_{\mathrm{A}}^{2}$ mais est égal à $\frac{\mathrm{I}}{4} \sigma_{\mathrm{A}}^{2}+\frac{\mathrm{I}}{4} \cdot \mathrm{R} \sigma_{\mathrm{A}}^{3}$ (Flock, I967; Hinkelmann, I969, I97I).

De même, la part de la variance totale due aux facteurs d'environnement communs aux individus d'une même portée, $c^{2}$, est estimée par :

$$
\frac{\widehat{\sigma}_{d}^{2}-\frac{\mathrm{I}-\mathrm{R}}{\mathrm{I}+\mathrm{R}} \cdot \hat{\sigma}_{s}^{2}}{\widehat{\sigma}_{\mathbf{P}}^{2}} \quad \text { plutôt que par } \frac{\widehat{\sigma}_{d}^{2}-\widehat{\sigma}_{s}^{2}}{\widehat{\sigma}_{\mathrm{P}}^{2}}
$$

( $\widehat{\sigma}_{d}^{2}$ est le composant de variance "entre mères ", tandis que $\widehat{\sigma}_{\mathrm{P}}^{2}$ est la variance phénotypique). En effet, si $\sigma_{s}^{2}$ s'est accru de $\frac{\mathrm{I}}{4} \mathrm{R} \cdot \sigma_{A}^{2}, \sigma_{d}^{2}$ s'est réduit de la même quantité.

Aucune correction n'est appliquée aux corrélations génétiques, $\gamma_{\mathrm{G}}$, pour $\mathrm{R} \neq \mathrm{O}$, car le facteur de correction figure à la fois au numérateur et au dénominateur et est, dès lors, sans effet.

L'écart-type de l'estimation de $h^{2}$ a été calculé d'après la formule proposée par Dickerson (1960) Pour $\mathrm{R} \neq 0$, cet écart-type de $h^{2}, \mathrm{~S}\left(h^{2}\right)$ subira la même correction que $h^{2}$.

Pour estimer l'écart-type d'une corrélation génétique, plusieurs méthodes ont été proposées (Mode et Robinson, I959; Tallis, I956; Robertson, i959 b; Scheinberg, ig66). La formule d'approximation proposée par RoBERTSON (1959 b) a été retenue pour sa simplicité. Toutefois, elle suppose que $\gamma_{\mathrm{G}}$ ne soit pas trop voisin de $\mathrm{I}$ et que la différence entre la corrélation " entre groupes " et la corrélation "dans les groupes " ne soit pas trop grande.

\section{3. - Correction des données}

Comme il a été dit plus haut, le matériel a été divisé en deux parties, correspondant aux deux types de ration utilisés : la première partie se rapporte à la période $6 \mathrm{I}-65$, la seconde partie à la période 66-70. Chacune de ces parties a fait l'objet d'une analyse particulière. Pour chacune d'elles, une correction des données a été faite : correction pour le sexe (à cause d'une répartition inégale; la correction n'est appliquée qu'au castrat), correction pour le poids abattu (tous les poids abattus sont ramenés à $72 \mathrm{~kg}$ ). Ces deux corrections ont été faites simultanément (régression multiple). Les sommes de carrés et sommes de produits des équations normales sont les sommes de carrés et sommes de produits "dans les portées ". On cherche ainsi à se soustraire à l'influence de tendances de toute nature. 


\section{III. - RÉSULTATS}

\section{I. - Résultats généraux et correction des données}

Dans le tableau 2, sont donnés, pour quatorze caractères et pour chacune des périodes, moyenne et écart-type (SP intra " années-stations »).

\section{TABLEAU 2}

Moyenne et écart-type phénotypique intra-année et station des caractères d'engraissement et de carcasse

\begin{tabular}{|c|c|c|c|c|}
\hline \multirow{2}{*}{ Caractères } & \multicolumn{2}{|c|}{ Période I (61-65) } & \multicolumn{2}{|c|}{ Période II $(66-70)$} \\
\hline & $\bar{x}$ & $\mathrm{~S}_{\mathrm{P}}$ & $\bar{x}$ & $\mathrm{~S}_{\mathbf{P}}$ \\
\hline Gain quotidien $(g) \ldots \ldots \ldots \ldots \ldots$ & 523 & 74 & 604 & 62 \\
\hline Indice consom. $(\mathrm{kg}) \ldots \ldots \ldots \ldots$ & 3,566 & 0,319 & 3,056 & 0,327 \\
\hline Rendement à l'abat. $(\%) \ldots \ldots \ldots$ & 79,71 & 1,45 & 80,18 & 1,40 \\
\hline Longueur carcasse $(\mathrm{cm}) \ldots$ & 74,30 & 1,83 & 73,00 & 1,95 \\
\hline Épais. moy. gras dors. $(\mathrm{cm}) \ldots \ldots$ & 2,70 & 0,37 & 2,20 & 0,39 \\
\hline (Tête + pieds) $/$ Poids abat. $(\%) \ldots$ & 6,67 & 0,44 & 6,46 & 0,46 \\
\hline Carré/Poids abattu $(\%) \ldots \ldots \ldots \ldots$ & 23,56 & 1,19 & 24,47 & 1,14 \\
\hline Jambon/Poids abattu $(\%) \quad \ldots \ldots \ldots$ & 23,91 & 1,16 & 24,64 & 1,17 \\
\hline Épaule/Poids abattu $(\%)$ & 15,51 & 0,75 & 15,69 & 0,75 \\
\hline Maigre total/Poids abat. $(\%)$ & 65,85 & 2,19 & 67,03 & 2,11 \\
\hline Lard dorsal/Poids abattu $(\%)$ & 6,13 & 1,22 & 6,03 & 1,16 \\
\hline Par. ventr./Poids abattu $(\%) \ldots \ldots$ & 16,25 & 1,03 & 15,83 & 1,13 \\
\hline Collier/Poids abattu $(\%) \ldots \ldots$ & 3,81 & 0,52 & 3,36 & $0,,^{\prime} 6$ \\
\hline Gras total/Poids abattu $(\%) \ldots$ & 27,55 & 2,28 & 26,55 & 2,27 \\
\hline
\end{tabular}

L'effet du sexe sur les caractères d'engraissement et de carcasse peut être évalué, d'après les valeurs du coefficient de régression partielle $\left(\beta_{1}\right)$ (régression du caractère sur le sexe, à poids abattu constant) figurant au tableau 3. Quel que soit le caractère envisagé, il n'y a pas de différence significative entre les deux périodes, en ce qui concerne la valeur du coefficient $\beta_{1}$. L'effet du sexe n'est pas significativement différent de zéro pour l'indice de consommation, le rendement à l'abattage. La lecture du tableau 3 fait apparaître, en outre, que le gain quotidien est légèrement plus élevé chez les castrats. Une carcasse de femelle est, en moyenne, plus longue $(+0,5 \mathrm{~cm})$ et plus maigre qu'une carcasse de castrat.

Les éléments d'une étude de l'influence des variations du poids abattu sur les caractères de carcasse et d'engraissement sont donnés au tableau 4 , où le coefficient de régression partielle, $\beta_{2}$, représente la variation du caractère pour une augmentation unitaire $(\mathrm{r} \mathrm{kg})$ du poids de la carcasse, à sexe constant. Ces coefficients sont comparables d'une période à l'autre; seuls, les coefficients relatifs au pourcentage de gras total dans la carcasse sont significativement différents, au seuil 5 p. Ioo. Ces coefficients ont généralement le signe attendu. Quand le poids de la carcasse aug- 
Effet du sexe sur les caractères d'engraissement et de carcasse

$\beta_{1}=$ différence castrat-femelle

$\mathrm{S}_{\beta_{1}}=$ écart type de $\beta_{1}$.

\begin{tabular}{|c|c|c|c|c|}
\hline \multirow{2}{*}{ Caractères } & \multicolumn{2}{|c|}{ Période I (61-65) } & \multicolumn{2}{|c|}{ Période II (66-70) } \\
\hline & $\beta_{1}$ & $S_{\beta_{1}}$ & $\beta_{1}$ & $s_{\beta_{1}}$ \\
\hline Gain quotidien (g) & $+29,269 * * *$ & 5,184 & $+35,059 * * *$ & 4,598 \\
\hline Indice consom. (g) & $+7,517$ & 26,089 & $-37,798$ & 20,483 \\
\hline Rendement à l'abat. $(\%)$.. & $-0,064$ & 0,137 & $+0,104$ & 0,098 \\
\hline Longueur carcasse $(\mathrm{cm}) \ldots \ldots \ldots$ & $-0,486^{* *}$ & 0,187 & $-0,572 * * *$ & 0,120 \\
\hline Épais. moy. gras dors. (cm) ..... & $+0,410 * * *$ & 0,037 & $+0,358^{* * *}$ & 0,024 \\
\hline (Tête + pieds) $/$ Poids abat. $(\%)$. & $-0,063$ & 0,044 & $-0,095^{* * *}$ & 0,031 \\
\hline Carré/Poids abattu $(\%) \quad \ldots \ldots \ldots$ & $-1,180^{* * *}$ & 0,095 & $-0,998^{* * *}$ & 0,073 \\
\hline Jambon $/$ Poids abattu $(\%) \ldots \ldots$ & $-0,929 * * *$ & 0,101 & $-0,888 * * *$ & 0,070 \\
\hline Épaule/Poids abattu $(\%) \ldots \ldots$ & $-0,328 * * *$ & 0,066 & $-0,258^{* * *}$ & 0,045 \\
\hline Maigre total/Poids abat. $(\%) \ldots$ & $-2,452 * * *$ & 0,149 & $-2,176 * * *$ & 0,115 \\
\hline Lard dorsal/Poids abattu $(\%) \ldots$ & $+1,428 * * *$ & 0,101 & $+1,359 * * *$ & 0,078 \\
\hline Par. ventr./Poids abattu $(\%) \quad \ldots$ & $+0,414 * * *$ & 0,096 & $+0,395 * * *$ & 0,067 \\
\hline Collier/Poids abattu $(\%) \ldots \ldots$ & $+0,227 * * *$ & 0,046 & $+0,234 * * *$ & 0,032 \\
\hline Gras total/Poids abattu $(\%) \ldots$ & $+2,461 * * *$ & 0,183 & $+2,319 * * *$ & 0,146 \\
\hline
\end{tabular}

N. B. : Seuil de signification : * $=0,05 ; \quad * *=0,01 ; \quad * * *=0,001$.

\section{TABLEAU 4}

Influence des variations du poids abattu sur les caractères d'engraissement et de carcasse $\beta_{2}=$ Coefficient de régression partielle du caractère sur le poids abattu (unité : I $\mathrm{kg}$ ) $\mathrm{S}_{\beta_{2}}=$ écart-type de $\beta_{2}$

\begin{tabular}{|c|c|c|c|c|}
\hline \multirow{2}{*}{ Caractères } & \multicolumn{2}{|c|}{ Période I (61-65) } & \multicolumn{2}{|c|}{ Période II (66-70) } \\
\hline & $\beta_{2}$ & $\mathrm{~S}_{\beta_{2}}$ & $\beta_{2}$ & $\mathrm{~S}_{\beta_{2}}$ \\
\hline Gain quotidien $(g) \ldots \ldots \ldots$ & $+6,410 * * *$ & 1,135 & $+5,720^{* * *}$ & 1,12 \\
\hline Indice consom. $(g) \ldots \ldots \ldots$ & $-9,78$ & 5,712 & $-18,230^{* * *}$ & 5,00 \\
\hline Rendement à l'abat. ( $\%)$ & $+0,364 * * *$ & 0,030 & $+0,327 * * *$ & 0,024 \\
\hline Longueur carcasse $(\mathrm{cm}) \ldots \ldots \ldots$ & $+0,122 * *$ & 0,041 & $+0,173^{* * *}$ & 0,029 \\
\hline Épais. moy. gras dors. $(\mathrm{cm}) \ldots$ & $+0,037$ & 0,008 & $+0,036$ & 0,006 \\
\hline (Tête + pieds) $/$ Poids abat. $(\%)$ & $-0,057^{* * *}$ & 0,010 & $-0,038 * * *$ & 0,007 \\
\hline Carré/Poids abattu $(\%) \quad \ldots \ldots \ldots$ & $-0,106 * * *$ & 0,021 & $-0,08 / 2 * *$ & 0,018 \\
\hline Jambon/Poids abattu $(\%) \ldots$ & $-0,123^{* * *}$ & 0,022 & $-0,124 * * *$ & 0,017 \\
\hline Epaule/Poids abattu $(\%) \ldots$ & $-0,065 * * *$ & 0,014 & $-0,093^{* * *}$ & 0,011 \\
\hline Maigre total/Poids abat. $(\%) \ldots$ & $-0,3 \mathbf{4} 0^{* * *}$ & 0,033 & $-0,373^{* * *}$ & 0,028 \\
\hline Lard dorsal/Poids abattu $(\%) \ldots$ & $+0,121 * * *$ & 0,022 & $+0,083^{* * *}$ & 0,019 \\
\hline Par, ventr./Poids abattu $(\%) \ldots$ & $+0,090 * * *$ & 0,021 & $+0,067^{* * *}$ & 0,016 \\
\hline Collier/Poids abattu $(\%) \quad \ldots$ & $+0,024$ & 0,010 & $+0,031 * * *$ & 0,008 \\
\hline Gras total/Poids abattu $(\%)$ & $+0,297 * * *$ & 0,040 & $+0,186^{* * *}$ & 0,036 \\
\hline
\end{tabular}

N. B. : Seuil de signification : * $=0,05 ; \quad * *=0,01 ; \quad * * *=0,001$. 
mente, la proportion de morceaux maigres diminte au profit de la proportion de morceaux gras. S'élèvent aussi, en même temps que le poids abattu, la longueur de la carcasse, le rendement à l'abattage, le gain quotidien. On s'attendait moins à ce que l'indice de consommation s'améliore avec une augmentation du poids à 1'abattage. Toutefois, le gain quotidien se trouve amélioré, et on sait qu'un gain quotidien élevé est favorable à l'indice de consommation.

\section{2. - Héritabilité}

Les valeurs du coefficient d'héritabilité $\left(h^{2}\right)$, de son écart-type $\left(\mathrm{S}_{h^{2}}\right)$ et du terme, $c^{2}$, représentant la part de la variance due aux effets d'environnement (plus les effets de dominance) communs aux sujets d'une même portée, figurent au tableau 5 . Ces estimations ont été corrigées pour la parenté entre truies accouplées au même verrat $(\mathrm{R}=0,05$ pour la période $\mathrm{I} ; \mathrm{R}=0,07$ pour la période $\mathrm{II})$. Les valeurs de $h^{2}$ se situent entre les limites 0,2 et 0,9 pour la période I, entre les limites 0,25 et 0,67 pour la période II. Si on s'en tient aux estimations de la période II, où l'écart-type est plus petit, on remarque que la plupart des estimations sont significativement différentes de zéro $\left(h^{2} \div \mathrm{S} h^{2}>2\right)$. Les caractères de carcasse, comme la longueur, l'épaisseur du lard dorsal, la proportion de morceaux maigres, la proportion de morceaux gras ont une héritabilité de 1'ordre de 0,5 à 0,6 . Le gain quotidien a une héritabilité relativement élevée de 0,6 alors que 1 'indice de consommation a une héritabilité inférieure à 0,4 dans les deux périodes.

Quant au terme $c^{2}$, sa valeur oscille autour d'une moyenne de l'ordre de o,Io.

TABLEAU 5

L'héritabilité $\left(h^{2}\right)$, son écart-type $\left(S h^{2}\right)$ et le terme $c^{2}$ (composante "nichée " de la variance totale) pour les caractères d'engraissement et de carcasse

\begin{tabular}{|c|c|c|c|c|c|c|}
\hline \multirow{2}{*}{ Caractères } & \multicolumn{3}{|c|}{ Période I (61-65) } & \multicolumn{3}{|c|}{ Période II $(66-70)$} \\
\hline & $h^{2}$ & $\mathrm{~S}_{h}{ }^{2}$ & $c^{2}$ & $h^{2}$ & $\mathrm{~S}_{h}^{2}$ & $c^{2}$ \\
\hline Gain quotidien & 0,203 & 0,272 & 0,310 & 0,632 & 0,217 & 0,051 \\
\hline Indice de consom. ... & 0,395 & 0,297 & 0,250 & 0,352 & 0,180 & 0,140 \\
\hline Longueur carcasse ......... & 0,898 & 0,304 & $-0,135$ & 0,533 & 0,222 & 0,168 \\
\hline Épais. moy. gras dors. . . . . . . & 0,203 & 0,224 & 0,149 & 0,653 & 0,207 & $-0,015$ \\
\hline (Tête + pieds)/Poids abat. .... & 0,175 & 0,200 & 0,091 & 0,252 & 0,134 & 0,014 \\
\hline Carré/Poids abattu ......... & 0,987 & 0,334 & $-0,107$ & 0,540 & 0,202 & 0,067 \\
\hline Jambon/Poids abattu $\ldots \ldots \ldots$ & 0,453 & 0,272 & 0,120 & 0,437 & 0,194 & 0,128 \\
\hline Épaule/Poids abattu ..... & 0,501 & 0,268 & 0,068 & 0,490 & 0,204 & 0,125 \\
\hline Maigre tot./Poids abattu . ..... & 0,928 & 0,355 & 0,018 & 0,595 & 0,219 & 0,100 \\
\hline Lard dorsal/Poids abattu ..... & 0,417 & 0,272 & 0,148 & 0,375 & 0,182 & 0,129 \\
\hline Par. ventr./Poids abattu ..... & 0,725 & 0,306 & 0,018 & 0,344 & 0,178 & 0,134 \\
\hline Collier/Poids abattu ......... & 0,469 & 0,221 & $-0,066$ & 0,671 & 0,222 & 0,039 \\
\hline Gras tot./Poids abattu ... & 0,914 & 0,343 & $-0,011$ & 0,507 & 0,200 & 0,092 \\
\hline
\end{tabular}

\section{3. - Corrélations}

Le tableau 6 est consacré aux corrélations phénotypiques $\left(r_{\mathrm{P}}\right)$ et génétiques $\left(r_{\mathrm{G}}\right)$ entre les caractères d'engraissement et de carcasse, pris deux à deux. 


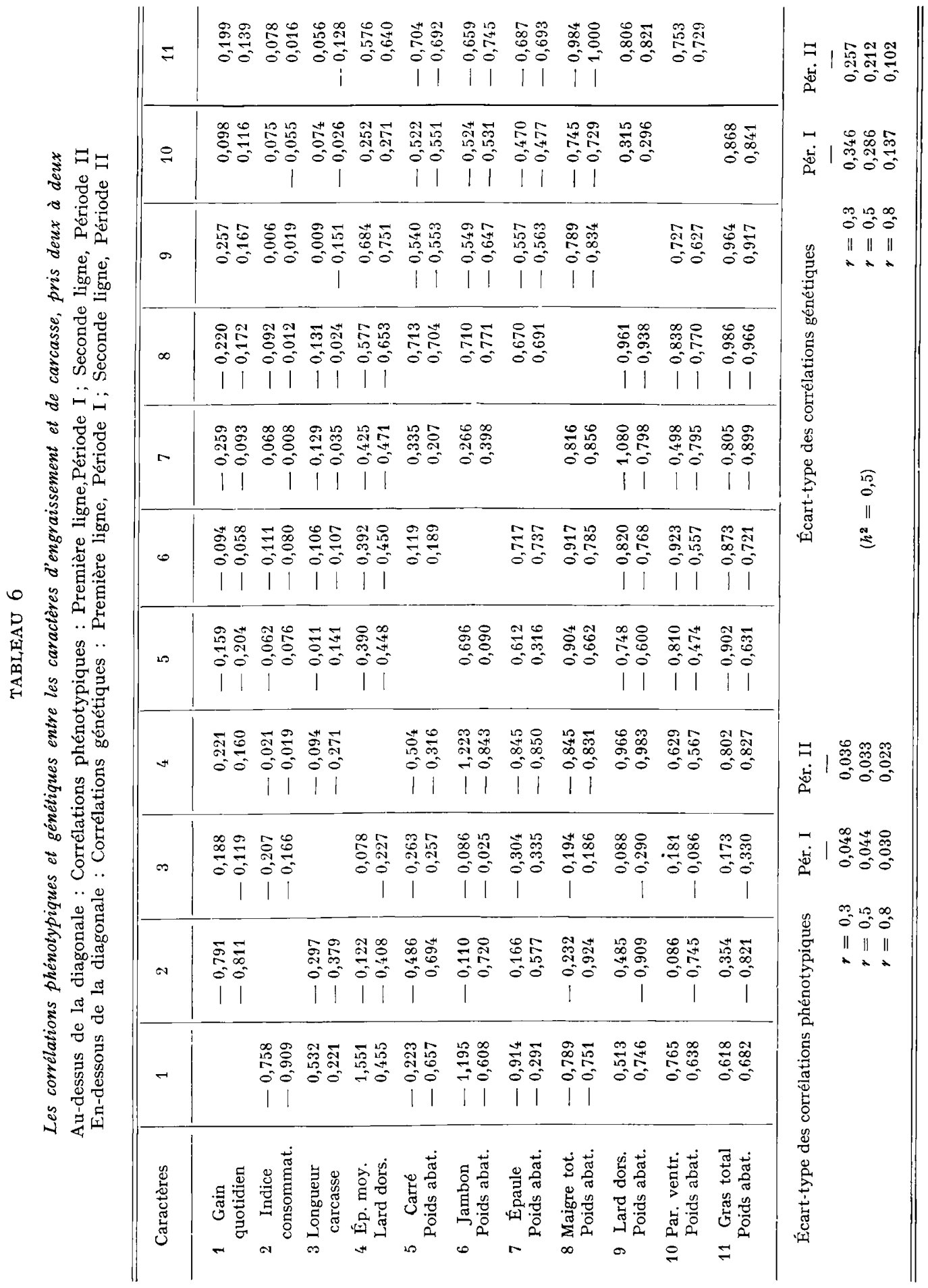


Les corrélations phénotypiques sont situées au-dessus de la diagonale, les corrélations génétiques en dessous de la diagonale. Pour chaque paire de caractère, la première valeur se rapporte à la période I, la seconde à la période II. En marge, figurent les écarts-types approximatifs de ces corrélations. Entre les deux caractères d'engraissement, gain quotidien et indice de consommation, la corrélation est élevée et de l'ordre de - o,8. Une croissance plus rapide implique un indice de consommation plus favorable. La corrélation phénotypique entre l'épaisseur moyenne du lard dorsal et la proportion de lard dorsal dans la carcasse est de 1'ordre de + 0,7. La corrélation génétique correspondante est de $+0,97$. Sa corrélation avec la proportion de morceaux maigres dans la carcasse est de l'ordre de - o,6o pour la corrélation phénotypique et de l'ordre de $-0,80$ pour la corrélation génétique. Une valeur aussi élevée souligne tout l'intérêt de la mesure de l'épaisseur du gras dorsal, pour la sélection individuelle des jeunes reproducteurs. Entre les différents segments de la découpe : carré, jambon, épaule, lard dorsal, lard ventral, les corrélations phénotypiques ont une valeur moyenne.

Les corrélations génétiques correspondantes ont une valeur plus élevée. L,es corrélations entre une partie et son tout sont élevées. (Ex. : corrélation entre carré et maigre total $r_{\mathrm{P}}=0,7 ; r_{\mathrm{G}}=0,75$; entre lard dorsal et gras total $r_{\mathrm{P}}=0,8 ; r_{\mathrm{G}}=0,93$ etc.)

Les corrélations phénotypiques entre, d'une part le gain quotidien, et d'autre part les caractères de carcasse, sont relativement faibles (de l'ordre de 0 , Io à 0,20 , en valeur absolue). Elles sont négatives lorsqu'il s'agit des morceaux maigres et positives lorsqu'il s'agit des morceaux gras. Le sens de ces corrélations est le même lorsqu'il s'agit des corrélations génétiques, avec cette différence que les corrélations génétiques sont plus élevées (avec la proportion de maigre total, $r_{\mathrm{G}}=-0,75$; avec la proportion de gras total, $\left.r_{\mathrm{G}}=+0,65\right)$.

L'indice de consommation est en corrélation moins nette avec les caractères de carcasse. Les corrélations phénotypiques sont faibles ; quant aux corrélations génétiques, plus élevées, elles sont positives dans une période et négatives dans l'autre. Pour la période I, l'indice de consommation est en corrélation négative avec la proportion de morceaux maigres et en corrélation positive avec la proportion de morceaux gras. Pour la période II, c'est l'inverse qui apparaît. Autrement dit, dans le premier cas, un indice de consommation plus favorable est lié à une proportion plus élevée de morceaux maigres alors que dans le second cas, un indice de consommation plus favorable est lié à une proportion plus élevée de morceaux gras. Peut-être ne s'agit-il là que de fluctuations d'échantillonnage autour d'une moyenne de population $\rho_{G}$, voisine de zéro. Toutefois, les corrélations se rapportant à la seconde période sont significativement différentes de zéro, ce qui n'est pas le cas pour celles de la période I.

Parmi les éleveurs de porc de Piétrain, de nombreuses controverses ont surgi quant à la longueur à préférer, dans le choix des animaux reproducteurs. On constate, dans le tableau des corrélations, une corrélation génétique positive avec le gain quotidien, une corrélation négative avec l'indice de consommation, une corrélation avec la proportion de morceaux maigres dont le signe est tantôt positif, tantôt négatif.

D'autre part, si on examine les moyennes annuelles des différents caractères, on observe, pour certains caractères de carcasse, en station 5 , une évolution continue (tabl. 7). Le tableau 7 donne, pour la période II (66-70), séparément par station, les 
moyennes annuelles des différents caractères. Les valeurs de ces moyennes sont les estimations des " effets principaux " dans une analyse à deux voies par les " moindres carrés " (année et sexe, nombres inégaux). L'interaction sexe $\times$ année n'a été significative que pour l'indice de consommation (station 5 , seuil $5 \mathrm{p}$. Ioo) et pour le pourcentage d'épaule dans la carcasse (station 2, seuil I p. IOO). En station 5, la tendance annuelle est hautement significative $(b \neq 0: \mathrm{P}<\mathrm{I} \mathrm{p}$ Ioo) pour la proportion de maigre total dans la carcasse (et par conséquent pour la proportion de morceaux gras). (Il ne s'agit évidemment pas d'une estimation de progrès génétique). Cette amélioration de la composition de la carcasse ne s'est accompagnée ni d'une diminution du gain quotidien, ni d'une détérioration de l'indice de consommation.

\section{TABLEAU 7}

Évolution annuelle des moyennes de différents caractères

(Ligne supérieure $=$ Station 2 ; ligne inférieure $=$ Station 5)

\begin{tabular}{|c|c|c|c|c|c|c|c|}
\hline \multirow{2}{*}{\multicolumn{2}{|c|}{ Caractères }} & \multicolumn{5}{|c|}{ Années } & \multirow{2}{*}{$\begin{array}{l}\text { Régression linéaire } \\
\text { de la moyenne } \\
\text { sur l'année }(b)\end{array}$} \\
\hline & & 1966 & 1967 & 1968 & 1969 & 1970 & \\
\hline Gain quotidien (g) & $\begin{array}{l}\text { St. } 2 \\
\text { St. } 5\end{array}$ & $\begin{array}{l}633 \\
560\end{array}$ & $\begin{array}{l}636 \\
632\end{array}$ & $\begin{array}{l}621 \\
652\end{array}$ & $\begin{array}{l}609 \\
627\end{array}$ & $\begin{array}{l}618 \\
662\end{array}$ & $\begin{array}{r}-5,6 \\
20,0\end{array}$ \\
\hline Indice consom. (kg) & $\begin{array}{l}\text { St. } 2 \\
\text { St. } 5\end{array}$ & $\begin{array}{l}3,191 \\
3,181\end{array}$ & $\begin{array}{l}3,060 \\
3,010\end{array}$ & $\begin{array}{l}3,044 \\
2,849\end{array}$ & $\begin{array}{l}3,341 \\
2,973\end{array}$ & $\begin{array}{l}3,211 \\
2,788\end{array}$ & $\begin{array}{r}0,032 \\
-0,082 \mathrm{P}<10 \%\end{array}$ \\
\hline Longueur carcasse $(\mathrm{cm})$ & $\begin{array}{l}\text { St. } 2 \\
\text { St. } 5\end{array}$ & $\begin{array}{l}73,5 \\
72,5\end{array}$ & $\begin{array}{l}72,7 \\
72,2\end{array}$ & $\begin{array}{l}7:, 7 \\
7: 2,0\end{array}$ & $\begin{array}{l}73,0 \\
72,9\end{array}$ & $\begin{array}{l}75,3 \\
73,6\end{array}$ & $\begin{array}{l}0,4 \\
0,28\end{array}$ \\
\hline Épais. lard dors. (mm) & $\begin{array}{l}\text { St. } 2 \\
\text { St. } 5\end{array}$ & $\begin{array}{l}25,0 \\
2 \div, 1\end{array}$ & $\begin{array}{l}26,0 \\
24,8\end{array}$ & $\begin{array}{l}27,0 \\
23,6\end{array}$ & $\begin{array}{l}25,0 \\
23,9\end{array}$ & $\begin{array}{l}25,0 \\
21,2\end{array}$ & $\begin{array}{l}-0,06 \\
-0,67\end{array}$ \\
\hline Carré/Poids abat. (\%) & $\begin{array}{l}\text { St. } 2 \\
\text { St. } 5\end{array}$ & $\begin{array}{l}23,73 \\
23,41\end{array}$ & $\begin{array}{l}23,70 \\
23,71\end{array}$ & $\begin{array}{l}23,63 \\
23,88\end{array}$ & $\begin{array}{l}23,94 \\
25,00\end{array}$ & $\begin{array}{l}23,95 \\
2{ }^{\prime}, 65\end{array}$ & $\begin{array}{l}0,37 \\
0,38\end{array}$ \\
\hline Jambon/Poids abat. ( $\%$ ) & $\begin{array}{l}\text { St. } 2 \\
\text { St. } 5\end{array}$ & $\begin{array}{l}23,29 \\
23,90\end{array}$ & $\begin{array}{l}24,06 \\
23,95\end{array}$ & $\begin{array}{l}24,29 \\
24,45\end{array}$ & $\begin{array}{l}24,01 \\
24,31\end{array}$ & $\begin{array}{l}23,62 \\
25,12\end{array}$ & $\begin{array}{l}0,06 \\
0,28 \mathrm{P}<5 \%\end{array}$ \\
\hline Epaule/Poids abat. $(\%)$ & $\begin{array}{l}\text { St. } 2 \\
\text { St. } 5\end{array}$ & $\begin{array}{l}15,51 \\
15,61\end{array}$ & $\begin{array}{l}15,95 \\
15,50\end{array}$ & $\begin{array}{l}15,27 \\
15,50\end{array}$ & $\begin{array}{l}15,07 \\
15,40\end{array}$ & $\begin{array}{l}15,0^{\prime} \\
15,60\end{array}$ & $\begin{array}{r}-0,18 \\
-0,01\end{array}$ \\
\hline Maigre tot./Pds abat. ( $\%)$ & $\begin{array}{l}\text { St. } 2 \\
\text { St. } 5\end{array}$ & $\begin{array}{l}64,79 \\
65,21\end{array}$ & $\begin{array}{l}65,92 \\
65,40\end{array}$ & $\begin{array}{l}65,45 \\
66,04\end{array}$ & $\begin{array}{l}65,09 \\
66,74\end{array}$ & $\begin{array}{l}64,83 \\
67,51\end{array}$ & $\begin{array}{l}-0,07 \\
0,59 \mathrm{P}<1 \%\end{array}$ \\
\hline Lard dorsal/Pds abat. $(\%)$ & $\begin{array}{l}\text { St. } 2 \\
\text { St. } 5\end{array}$ & $\begin{array}{l}7,07 \\
6,85\end{array}$ & $\begin{array}{l}7,21 \\
7,09\end{array}$ & $\begin{array}{l}6,99 \\
6,43\end{array}$ & $\begin{array}{l}6,91 \\
6,43\end{array}$ & $\begin{array}{l}6,99 \\
5,66\end{array}$ & $\begin{array}{l}-0,05 \\
-0,30 \mathrm{P}<5 \%\end{array}$ \\
\hline Par. ventr./Pds abat. $(\%)$ & $\begin{array}{l}\text { St. } 2 \\
\text { St. } 5\end{array}$ & $\begin{array}{l}16,42 \\
16,25\end{array}$ & $\begin{array}{l}15,94 \\
16,14\end{array}$ & $\begin{array}{l}15,97 \\
16,06\end{array}$ & $\begin{array}{l}16,52 \\
15,57\end{array}$ & $\begin{array}{l}16,43 \\
15,71\end{array}$ & $\begin{aligned} & 0,06 \\
- & 0,16 \mathrm{P}<5 \%\end{aligned}$ \\
\hline Gras tot./Pds abat. (\%) & $\begin{array}{l}\text { St. } 2 \\
\text { St. } 5\end{array}$ & $\begin{array}{l}28,90 \\
28,52\end{array}$ & $\begin{array}{l}27,72 \\
28,32\end{array}$ & $\begin{array}{l}28,15 \\
27,59\end{array}$ & $\begin{array}{l}28,31 \\
26,74\end{array}$ & $\begin{array}{l}28,37 \\
25,78\end{array}$ & $\begin{array}{l}-0,04 \\
-0,71 \mathrm{P}<1 \%\end{array}$ \\
\hline
\end{tabular}

D'autre part, une amélioration de la composition de la carcasse paraît aller de pair avec une modification de l'importance relative des différents morceaux. Nous avons étudié les rapports : carré/maigre total; jambon/maigre total ; épaule/maigre total ; lard dorsal/gras total ; parois ventrales/gras total. Dans la carcasse de femelle 
l'importance relative du carré et du jambon est plus élevée (tab1. 8). Chez le castrat, l'importance de l'épaule est plus grande. Pour les morceaux gras, la part relative des parois ventrales est sensiblement plus grande chez la femelle que chez le castrat. Ainsi, l'épaule est relativement plus développée chez le castrat. Toutefois, l'épaule, comme le jambon, mais à la différence du carré, est recouverte d'une couche relativement épaisse de tissu adipeux. On ne peut pas dire si cet effet du sexe porte sur une plus grande accumulation de graisse au niveau de l'épaule ou sur un développement plus marqué des muscles de 1'épaule.

\section{TABLEAU 8}

Influence du sexe sur l'importance relative des différents morceaux de la carcasse

(Période II : 1966-r970)

$\beta_{1}=$ différence castrat-femelle

$\mathrm{S}_{\beta_{2}}=$ écart-type de $\beta_{1}$

\begin{tabular}{l|c|c}
\hline \multicolumn{1}{c|}{ Caractères } & $\beta_{1}$ & $\mathrm{~S}_{\beta_{1}}$ \\
\hline Carré/maigre total $\ldots \ldots \ldots \ldots$ & $-0,294^{* * *}$ & 0,082 \\
Jambon/maigre total $\ldots \ldots \ldots \ldots$ & $-0,137$ & 0,075 \\
Épaule/maigre total ....... & $0,398^{* * *}$ & 0,054 \\
Lard dorsal/gras total....... & $2,913^{* * *}$ & 0,194 \\
Parois ventr./gras total ...... & $-3,441^{* * *}$ & 0,209 \\
\end{tabular}

N. B. : Seuil de signification : $*=0,05 ; \quad * *=0,01 ; * * *=0,001$.

Nous avons observé, plus haut, une amélioration de la proportion de morceaux maigres dans la carcasse. Parallèlement, nous observons une diminution significative du rapport épaule/maigre total $\left(b=-0,23^{* *}\right)$ et une élévation significative du rapport parois ventrales/gras total $\left(b=0,9 I^{*}\right)$. Il n'y avait pas non plus d'interaction sexe $x$ année significative.

\section{DISCUSSION}

Préalablement aux analyses de variance et de covariance proprement dites, la plupart des auteurs effectuent une correction des données.

KING (I957) pratique une correction " intra portées " pour le sexe et le poids abattu. Smith et al. (I962) ne retiennent pour leurs analyses que les lots équilibrés ( 2 castrats +2 femelles). Ils traitent les sexes séparément et corrigent les données pour la station, la période (périodes de six mois), le dernier poids vif. Smith et al. (I965) ne retiennent que les lots équilibrés et corrigent pour la station et la période (périodes de trois mois), pour l'âge au début du contrôle et le dernier poids vif. Une correction pour la période de l'année et le poids à l'abattage est appliquée par Jonsson et KING 
(I962). Comme les données étaient "balancées » en ce qui concerne le sexe, aucune correction ne fut appliquée pour les différences dues au sexe. Jonsson (I963 et I965) divise son matériel en sous-groupes définis par : année station, sexe. Dans chaque sous-groupe, il applique une correction pour la période de l'année (soit le mois, soit le trimestre) et le poids de la carcasse pris 24 heures après l'abattage. Dans son étude sur le Large White français, OL,IVIER (I970) calcule, séparément par sexe, les facteurs de correction (coefficients de régression partielle) pour le poids initial, l'âge initial et le poids vif à l'abattage. FLOCK (I970) transforme chaque donnée en son écart par rapport à une moyenne mobile intra-station (période de deux mois) et analyse chaque sexe séparément. Dans notre étude, nous exprimons la valeur pondérale de chaque morceau de la découpe en $\mathrm{p}$. roo du poids de la carcasse.

Nous aurions pu utiliser les poids des différents morceaux, corrigés pour un même poids abattu. Les résultats ne s'en trouveraient pas affectés. Les coefficients d'héritabilité et les coefficients de corrélation sont pratiquement les mêmes, qu'ils se rapportent au pourcentage corrigé pour le poids abattu ou au poids corrigé pour le poids abattu. En outre, les corrélations génétiques entre ces deux modes d'expression sont pratiquement égales à l'unité.

Le sexe paraît avoir le même effet dans toutes les races : la femelle est plus longue et sa carcasse est plus riche en morceaux maigres. En ce qui concerne la longueur, par exemple, Smiтh et al., (I962) trouvent une différence de $0,7 \mathrm{~cm}$, chez le British Large White (pubis, Ire côte) ; chez le Landrace danois, Jonsson (I965) trouve une différence de $0,6 \mathrm{~cm}$ (pubis, atlas) et OLLIVIER (1970), chez le Large White français, une différence de $\mathrm{I}, 3 \mathrm{~cm}$ (pubis, atlas), Jonsson (I965) remarque, en outre, que, avec la sélection, la différence entre les sexes pourrait s'atténuer, ce qui implique, en quelque sorte, une réduction de l'effet de la castration.

En ce qui concerne l'estimation des paramètres génétiques, notre échantillon analysé n'avait sûrement pas la taille désirée. Les écarts-types des paramètres sont particulièrement sensibles à la taille du matériel analysé. TALLIS et KLOSTERMAN (I959) et ROBERTSON (I959 $a$ ) ont étudié le problème d'une estimation efficiente de 1'héritabilité. D'après RoBERTson (I959 a), la meilleure estimation de la corrélation paternelle, en vue de l'estimation de $h^{2}$, est obtenue pour un descendant par mère et un nombre de descendants par père de 1'ordre de $\mathrm{I} / t$ où $t$ est la corrélation intraclasse (valeur attendue : I/ $/ h^{2} / 4$ ). Si les corrélations paternelle et maternelle ont la même valeur, une information également optimale, sur ces deux coefficients, implique la structure suivante : 3 à 4 mères par père et $I / 2 t$ descendants par mère.

La structure optimale n'est pas différente lorsqu'il s'agit d'estimer les coefficients de corrélation génétique (ROBERTSON, I959 b). Il n'en reste pas moins vrai qu'il reste beaucoup à faire pour améliorer les estimations de 1'héritabilité, des corrélations phénotypiques et génétiques.

Les erreurs d'échantillonnage de la corrélation génétique sont si élevées, avec les méthodes dont on dispose actuellement (surtout lorsque l'héritabilité est faible) que la perspective de mesurer ces paramètres avec précision n'est guère favorable (LUSH, I966). Une corrélation génétique entre caractères peut être due : Io à un linkage entre gènes, $2^{\circ}$ à une association fortuite entre gènes, $3^{\circ}$ à $1^{\prime}$ action pléïotropique des gènes. Dans une grande population panmictique en équilibre, les éventualités I et 2 ne doivent pas être prises en considération. Une race, où la sélection est forte, ne peut être assimilée à une grande population. Dans ce cas, une associa- 
tion fortuite entre gènes ne peut être exclue sachant, néanmoins, qu'elle ne sera pas durable.

D'une revue des estimations des corrélations génétiques entre caractères d'engraissement et caractères de carcasse, HAZEL, (I G63) note qu'on peut commencer à tirer des conclusions, néanmoins sujettes à révision, concernant le sens et la grandeur de ces corrélations, tant l'écart-type d'une corrélation génétique est grand surtout lorsque cette corrélation est faible.

L'élaboration d'un index de sélection requiert qu'on dispose, au préalable, d'estimations valables des paramètres génétiques (héritabilités, corrélations génétiques) concernant les caractères mesurés soit sur l'animal vif, soit sur la carcasse.

Les données en provenance des stations de testage représentent un matériel assez artificiel en ceci que les animaux proviennent de parents choisis et que les descendants d'un même verrat peuvent avoir connu un même milieu avant le test.

On pouvait se demander si les estimations des coefficients d'héritabilité et des coefficients de corrélation génétique calculées lors d'expériences de sélection (héritabilité et corrélation génétique réalisées : FALCONER, I960) n'auraient pas plus de valeur que les estimations obtenues par les méthodes habituelles. Ce problème a été abordé par Hris (I97I). Il a montré que, pour un même nombre d'animaux contrôlés, du moment qu'on a utilisé le plan optimum approprié, les estimations d'héritabilités réalisées sont meilleures; malheureusement, elles prennent beaucoup plus de temps. En ce qui concerne les corrélations génétiques, cet auteur arrive à la conclusion que les plans d'expérience optimum pour l'estimation des héritabilités réalisées pourront convenir pour l'estimation des corrélations génétiques réalisées; en outre, comme les expériences de sélection s'avèrent efficaces pour estimer les héritabilités, elles doivent 1'être également pour l'estimation des corrélations génétiques. Néanmoins, pour atteindre leur but, de telles expériences de sélection doivent comporter un nombre relativement élevé d'animaux.

Dans la présente étude, la concordance entre les deux périodes, en ce qui concerne les corrélations, est meilleure pour les corrélations phénotypiques que pour les corrélations génétiques. Cette observation est attendue, étant donné l'écart-type élevé de ces derniers.

Les corrélations génétiques sont généralement supérieures aux corrélations phénotypiques correspondantes et lorsque le matériel analysé est de grande taille, il y a une bonne concordance entre les unes et les autres. Ainsi, Smith et al. (I965) trouvent une corrélation de 0,86 entre les corrélations phénotypiques et les corrélations génotypiques, pour les 26 caractères étudiés. Ce résultat suggère à ces auteurs qu'en l'absence d'estimations valables des corrélations génétiques, on pourrait se contenter de les estimer à partir des corrélations phénotypiques. Ils pensent que les écarts entre les corrélations génétiques et phénotypiques, lors d'analyses particulières, sont à mettre sur le compte d'erreurs d'échantillonnage.

La corrélation entre le gain quotidien et l'indice de consommation est élevée, qu'il s'agisse de la corrélation phénotypique ou de la corrélation génétique. Faisant la moyenne de huit études, SMITH et al. (I965) trouvent une corrélation génétique $\mathrm{de}-0,76$. Pour les périodes I et II, nous trouvons respectivement $-0,76$ et $-0,9 \mathrm{I}$. Il s'agit, dans tous ces cas, d'une alimentation restreinte. On peut prévoir que cette corrélation soit plus faible, lors d'alimentation ad libitum (OWEN et MORTON, I969). On se souviendra que, dans cette corrélation, il y a une part d'automaticité, le numé- 
rateur du rapport donnant le gain quotidien étant le dénominateur du rapport donnant l'indice de consommation (SuTHERLAND, I965).

La longueur de la carcasse est en corrélation génétique positive avec le gain quotidien (+ 0,I4 moyenne de huit études, Smi'r et al., I965; + o,22 dans la présente étude, période II) ; en corrélation génétique négative avec l'indice de consommation (- o, 08 moyenne de huit études, Surre et al. I965; - o,38, présente étude, période II) et avec l'épaisseur moyenne du lard dorsal (- $-0,30$ moyenne de huit études ; - 0,23 présente étude, période II). Les corrélations entre la longueur et les pourcentages de morceaux maigres et de morceaux gras sont de signe opposé selon que l'on envisage la période I ou la période II. Dans la période I, les porcs plus longs sont plus gras; tandis que dans la période II, c'est l'inverse qu'on constate. Smirn et al. (I965) relèvent une corrélation négative entre la longueur de la carcasse et la surface de 1'ilio-spinal ( $-0,08$, moyenne de huit études).

Un gain quotidien élevé est régulièrement associé à une carcasse plus grasse (épaisseur moyenne du lard dorsal, pourcentage des morceaux gras). Par contre, la moyenne de huit études donne une corrélation génétique de $-0,15$ entre le gain quotidien et l'épaisseur du gras dorsal (Smirh et al., I965).

Quant à la relation entre l'indice de consommation et la composition de la carcasse, nous obtenons des résultats contradictoires, un bon indice de consommation étant associé à une carcasse maigre, dans la période $\mathrm{I}$, à une carcasse grasse dans la période II. Pour les deux périodes, nous trouvons une corrélation génétique négative entre l'indice de consommation et l'épaisseur moyenne du lard dorsal, autrement dit une consommation plus élevée par $\mathrm{kg}$ de croît est associée à une épaisseur de lard dorsal plus faible. Par contre, Sмmтr et al. (I965) trouvent une corrélation génétique positive entre l'indice de consommation et l'épaisseur moyenne du gras dorsal : $+0,44(+0,2 \mathrm{I}$ moyenne de huit études).

Ainsi, au vu des résultats que nous obtenons dans cette étude, nous constatons, chez le Piétrain, quelques différences en ce qui concerne le sens des relations qu'entretiennent les caractères d'engraissement d'une part et les caractères de carcasse d'autre part.

A la différence de ce que SMirh et al. (I965) dégagent d'une revue de huit études, et de ce que FLock (I970) observe chez le Landrace allemand, chez le Piétrain un gain quotidien plus élevé et un meilleur indice de consommation (ces deux choses allant de pair) sont associés à une carcasse plus grasse. En outre, celle-ci pourrait être, en moyenne, légèrement plus longue.

Les variations annuelles que nous avons rapportées pour la période II ont fait apparaitre une amélioration de la composition de la carcasse, sans détérioration ni du gain quotidien, ni de l'indice de consommation. Remarquons que les analyses de variance et de covariance se font "intra-années ". D'autre part, la corrélation génétique entre caractères pourrait être négative alors que la corrélation due à l'environnement serait positive.

Nous n'avons pas retrouvé la forte interaction sexe $\times$ année-station observée par Jonsson pour le gain quotidien et l'indice de consommation. L'amélioration de la composition de la carcasse, observée en station 5, a été parallèle dans les deux sexes. Cette absence d'interaction sexe $\times$ année pourrait suggérer l'absence d'une interaction sexe $\times$ génotype, s'il s'avérait que l'amélioration est, pour une bonne part, de nature génétique. 
Des auteurs ne trouvent pas de différence significative entre les sexes en ce qui concerne les coefficients d'héritabilité. Mais, il est vrai que l'écart-type est élevé ; de là, le faible pouvoir discriminant de pareille comparaison (SMITH et al., I962, SMITH et al., I965). Toutefois, FREDEEN et JONSSON (I957) trouvent une différence significative entre les sexes pour les variances géniques du gain quotidien et de l'indice de consommation.

L'héritabilité de la note pour la couleur de la viande dans la section du muscle Longissimus dorsi est significativement différente de zéro chez les femelles, mais non chez les castrats (JoNSSON, I965)

Comparant les estimations des coefficients de corrélation obtenues dans les deux sexes, Smi't et al. (r965) trouvent une corrélation de o,96 entre les corrélations phénotypiques et une corrélation de $0,6 \mathrm{I}$ entre les corrélations génétiques. Ces auteurs ont mis en évidence une interaction sexe $\times$ verrat significative pour neuf caractères dont le gain quotidien, l'épaisseur du gras dor:al, la longueur de la carcasse, la surface de l'ilio-spinal. Cette interaction pouvait résulter soit de différences entre les sexes pour le composant de variance paternel $\left(\sigma_{s}^{2}\right)$, soit de différences dans le classement des verrats selon que celui-ci est basé sur la performance des descendants mâles ou sur la performance des descendants femelles. Dans le cas du matériel étudié par SMITH et al., les effets d'interaction étaient surtout dus à une différence de classement des verrats (corrélations génétiques entre les performances des deux sexes significativement plus petites que l'unité).

Comparant des porcs nourris ad libitum et des porcs de même portée rationnés (la restriction a été de I3 p. IOo chez les femelles et de 9 p. IOO chez les castrats), MINKEMA (I970) trouve une interaction sexe $\times$ niveau significative, pour les caractères d'engraissement (gain quotidien, indice de consommation), mais non pour les caractères de carcasse. Analysant les données des stations de progeny-test, cet auteur a trouvé une interaction verrat $\times$ sexe pour la proportion de jambon dans la carcasse.

Reçu pour publication en février 1972.

\section{REMERCIEMENTS}

Les auteurs tiennent à remercier pour leur précieuse collaboration M. l'Ingénieur R. CAMERLynck, Conseiller de Zootechnie de l'État et M. Kieffer et Laplanche du Centre de Calcul et de Traitement de 1'Information de 1'Université de Liège. Ils adressent également leurs remerciements à M. J.-J. Lauvergne, C. Legault et L. Ollivier (I. N. R. A., Jouy-en-Josas) pour leurs remarques et suggestions à la lecture du manuscrit.

\section{SUMMARY}

THE GENETIC PARAMETERS OF FATTENING AND

CARCASS TRAITS IN PIÉTRAIN PIG

The test results of $\mathrm{x}$ 087 Pietrain pigs from 120 boars and $3 \mathbf{1} 7$ sows are analyzed.

The effects of sex and of variations in slaughter weights on the fattening and carcass traits are studied. The genetic parameters, heritabilities and genetic correlations, are estimated for I 3 traits. 
The genetic correlation between daily gain and meat percent in the carcass is negative and significantly different from zero.

The observed trend in the character means has also been studied.

A significant increase is observed for the percent lean, in one station. This improvement is the same for the females as for the castrated males (zero sex $\times$ year interaction).

During the same period, the relative importance of the shoulder among the lean cuts has decreased.

\section{RÉFÉRENCES BIBLIOGRAPHIQUES}

De Wilde R., Vanschoubroek F., I97o. Vergelijking van het voeder van de Belgische varkensselektie mesterijen in twee verschillende bedrijfsomstandigheden. Vlaams. Dierg. Tijdschr., 39, 489-499.

Dickerson G. E., I960. Techniques for research in quantitative animal genetics, 56-105. Techniques and procedures in animal production research. Amer. Soc. Anim. Prod.

FALCONER D. S., I96o. Introduction to Quantitative Genetics. Ronald. New York.

FLock D., x967. Berechnung der durchschnittlichen Verwandtschaftsgrades aus der Haüfigkeit von Geschwister Paaren. Z. Tierz. Zücht Biol., 63, 235-239.

FLock D. K., I970. Genetic parameters of German Landrace pigs estimated from different relationships. J. Anim. Sci., 30, 839-843.

FREDEEN H. T., JonsSon P., 1957. Genic Variance and Covariance in Danish Landrace Swine as evaluated under a system of individual feeding of progeny-test groups. Z. Tierz. Z Zïcht Biol., 70, 348-363.

Hanset R., I97r. Étude des variations de la composition de la carcasse, de la valeur commerciale et du revenu, chez le porc Piétrain. Ann. Méd. vét., 115, 285-305.

Hazel L. N., r943. The Genetic Basis for constructing Selection Indexes. Genetics, 28, 476-490.

Hazel L. N., 1963. Genetic problems in breeding meat type pigs. Eur. As. Anim. Prod., Rome.

Hill W. G., r97r. Design and efficiency of selection experiments for estimating genetic parameters. Biometrics, 27, 293-3Ir.

HinkELMAN K., I969. Estimation of heritability from experiments with related dams. Biometrics, 25, 755 .

Hinkelman K., I97I. Estimation of heritability from experiments with inbred and related individuals. Biometrics, 27, 183 .

Jonsson P., x963. Danish pig progeny testing results. Z. Tierz. Ziucht Biol., 76, 205-252.

Jonsson P., 1965. Analysis of characters in the Danish Landrace pig. In 350 beretn. fra forsøgslab. København.

KEMPTHORNE O., r954. The correlation between relatives in a random mating population. Proc. Roy. Soc. (B), 143, гоз-гі3.

Kempthorne O., 1957. An Introduction to Genetic Statistics. Wiley.

KING J. W. B., I957. The heritability of carcass traits in british bacon pigs. Proc. Brit. Soc. Anim. Prod. ., 49-69.

KING S. C., Henderson C. R., 1954. Variance components analysis in heritability studies. Poult. Sci., 33, I47-I54.

I.E Roy H. L., I96o. Statistische Methoden der Populations Genetik. Birkhäuser.

LE Roy H. L., I966. Elemente der Tierzucht. Bayerischer Landwirtschafts Verlag, München.

Lush J. L., I966. Ways to improve a population genetically. Proc. 3rd Cong. S. Afr. Genet. Soc. (Pretoria), II-I6.

Malécot G., r948. Les Mathématiques de l'Hérédité. Masson. Paris.

Minkema D., x970. A preliminary report on the interaction between genotype, feeding level and sex in Dutch Landrace pigs. Paper presented at the meeting of the E. A. A. P., Budapest. I970.

Mode C. J., Robinson H. F., I959. Pleiotropism and the genetic variance and covariance. Biometrics, 15, 518-537.

Ollivier L., r97o. L'épreuve de la descendance chez le porc Large White français de 1953 à I966. I. Analyse de la variation. Ann. Génét. Sél. anim., 2, 3I I-324.

Owen J. B., Morton J. R., I969. The association of food conversion ratio, age at slaughter and carcass quality in pigs fed ad libitum Anim. Prod., 11, 317-324.

Robertson A., r959a. Expérimental design in the evaluation of genetic parameters. Biometrics, 15, 219-226.

RoBertson A., r959 b. The sampling variance of genetic correlation coefficient. Biometrics, 15, 469485 .

Scheinberg E., I 966 . The sampling variance of the correlation coefficients estimated in genetic experiments. Biometrics, 22, 187-191. 
Sмiтн C., I962. Estimation of genetic change in farm livestock using field records. Anim. Prod., 4, 239-25I.

Smith C., King J. W. B., Gilbert N., 1962. Genetic parameters of British Large White Bacon pigs. Anim. Prod., 4, 128-143.

Sutherland T. M., I965. The correlation between feed efficiency and rate of gain, a ratio and its denominator. Biometrics, 21, 739-740.

TALLIS G. M., 1959. Sampling errors of genetic correlation coefficients calculated form the analyses of variance and covariance. Austr. J. Stat., 1, 35-43.

Tallis G. M., Klosterman E. W., I959. Efficient estimates of heritability from paternal half-sib correlations. J. Anim. Sci., 18, 622-628.

Van Snick G., Camerlynck R., ig66. Le porc Piétrain. World Rev. Anim. Prod., 3, 95-III.

WrIGHT S., r922. Coefficients of inbreeding and relationship. Amer. Nat., 56, 330-338. 